\title{
Analysis of ZN-Stained Sputum Smear Enhanced Images for Identification of Mycobacterium Tuberculosis Bacilli Cells
}

\author{
Jadhav Mukti \\ Institute of Management Studies \\ \& Information Technology Aurangabad,
}

\author{
Kale K.V. \\ Department of Computer Science \& IT, \\ Dr. BAM University, Aurangabad,
}

\begin{abstract}
The analysis of ZN-Stain sputum smear images for the proper identification of Mycobacterium tuberculosis. For controlling on TB the early detection of TB is important this detection of $\mathrm{Tb}$ through microscope, in low- and middle-income countries. ZN-Staining commonly used for detecting M.TB bacilli from sputum. Tuberculosis (TB) diagnosis by manual observation varies depending on the quality of the smear and skill of the pathologist .This manual screening method is time consuming, tedious \& sometime it may confusing with some Non tuberculosis bacilli or some rod shape stain residue. Due to this we gets the faulty results. For this reason need of atomization is required. We present an enhancement method on images of Ziehl-Nelson stained sputum smears obtained using a bright field microscope. Some time the original images are noisy, degrade or blurred it need to preprocessing, enhancing the image for better result. Many of the scientist worked on gray level images or some used color images. We compare contrast stretching (High contrast) technique with sharping linear spatial filter by unsharp masking for improving the quality of ZN-Stained Sputum Smear image. The contrast stretching gives better results than the sharping image by linear spatial unsharp masking.
\end{abstract}

\section{Keywords}

Enhanced images, Mycobacterium Tuberculosis (M.TB), Acid Fast Bacilli (AFB), Contrast stretching, ZN-Stained (Ziehl-Neelsen), Sharpening image.

\section{INTRODUCTION}

Tuberculosis is a chronic infectious disease that is transmitted by cough-propelled droplets that carry the with Mycobacterium tuberculosis(M.TB). Tuberculosis (TB) remains one of the leading causes of death. According to recent data shown by WHO in 2008 , there were an estimated 8.9-9.9 million incident (number of new and relapse cases of TB in year) cases of TB, 9.6-13.3 million prevalent(number of TB cases (all forms) at a given point in time) cases of TB, 1.1-1.7 million deaths from TB among HIV-negative people and an additional $0.45-0.62$ million TB deaths among HIVpositive people (classified as HIV deaths in the International Statistical smear microscopy with the Ziehl-Neelsen Classification of Diseases), with best estimates of 9.4 million, 11.1 million, 1.3 million and 0.52 million, respectively[1]. TB may affect many body organs but primarily targets the lungs[2].
The main means of diagnosis and treatment of TB in lowand middle-income countries is technique, due to its simplicity, rapidity, reproducibility, low cost, and effectiveness in detecting infectious cases [3]. However manual screening technique is time consuming, laborintensive, tedious it required experienced technologist [4]. The sputum specimen that has undergone the process of staining using Ziehl-Neelsen procedure it make mycobacterium $\mathrm{Tb}$ cells appear red and other sputum cells appear blue background after adjusting the slide under 100x objective lens. These images are captured by digital camera as input to the work.

\section{LITERATURE SURVEY}

Several methods are in use that may increase the speed or sensitivity of microscopy somewhat. However, the quality of diagnosis depends on the conditions under which it is commonly performed: poor equipment, heavy workload, and inexpert \& unskilled staff.

Several authors have addressed segmentation of bacteria particle. Verpoulos et al.[4][5].Worked on gray level images used an identification method based on shape descriptors and Neural network classifiers showing a sensitivity(ratio of true positive decision against the total number of positive cases) of $94.1 \%$. Wilkinson [6] proposed a rapid multiresolution segmentation technique based on computing different thresholds for different areas of a grey level image. Other authors use the color information as the key discriminate factor either for bacteria segmentation and identification[7][8].

Manuel G. Forero et al.[9-11] proposed a technique of bacilli segmentation based on chromatic information, a multi thresholding image segmentation techniques and simple color filtering the second approach based on the use of gray-level morphological operators only to the green channel, the third approach is bacilli detection based on heuristic acknowledge extracted extracted from the bacilli shape contour. It uses also the color information for image segmentation and finally a classification tree is used to categorize if a sample is positive or negative. R.A.A. Raof et al.[12] proposed the thresholding procedures involved setting of boundaries based on grey values or intensities of image pixels. Hue color component based approach is proposed to segment the bacilli by adaptive choice of the hue range[13].

All above the works are carried on the microscopic images either these images are acquired directly by adjusting digital camera in eyepiece of microscope or by CCD camera. The 
acquired images of $\mathrm{ZN}$-sputum smear are blurred, noisy, degraded, having low resolution. Thus it needs to enhance the image for proper identification of TB bacilli from other sputum tissue stain residue.

\section{SOURCE DATA}

The data Acquisition for this work are capture images of $\mathrm{ZN}$ Stained sputum smear (Ziehl -Neelsen / Acid Fast Stain sputum smear slide) at $100 \mathrm{X}$ magnification by using Digital camera 7.4 MP. And saved in to bitmap (*.bmp) format.

Enhancement is to process an image so that the result is more suitable than the original image for a specific application. Here the techniques used for enhancement are spatial domain methods. The term special domain approach mean direct manipulation of pixels in an image. Techniques used for enhancement of images:-

1) Contrast stretching

2) Unsharp masking

\section{CONTRAST STRETCHING}

It is simplest piecewise linear function Contrast stretching transformation also known as normalization. Low contrast images can result from poor illumination, lack of dynamic range in the imaging sensor, or wrong setting of lens aperture during image acquisition. It is a simple image enhancement technique which attempts to improve the contrast in an image by 'expanding' the range of intensity values[15].

Contrast stretching makes sure the input sample with the lowest value is mapped to black; and that the one with the highest to 1.0. The values are recalculated by using linear interpolation. Contrast stretching automatically counters under and over exposure, as well as the ability to extend the used luminance range.

Before the stretching is to be performed it is necessary to specify the upper and lower pixel value limits over which the image is to be normalized. Often these limits will just be the minimum and maximum pixel values that the image type concerned allows. For example for 8-bit graylevel images the lower and upper limits might be 0 and 255. Call the lower and the upper limits a and $\mathrm{b}$ respectively[16].

The simplest sort of normalization then scans the image to find the lowest and highest pixel values currently present in the image. Call these $c$ and $d$. Then each pixel $P$ is scaled using the following function

$$
\text { Pout }=(\text { Pin }-c)\left(\frac{a-b}{d-c}\right)+a \text { Eq...(1) }
$$

Values below 0 are set to 0 and values about 255 are set to 255.

The problem with this is that a single outlying pixel with either a very high or very low value can severely affect the value of $c$ or $d$ and this could lead to very unrepresentative scaling. Therefore a more robust approach is to first take a histogram of the image, and then select $c$ and $d$ at, say, the 5th and 95th percentile in the histogram (that is, $5 \%$ of the pixel in the histogram will have values lower than $c$, and $5 \%$ of the pixels will have values higher than $d$ ). This prevents outliers affecting the scaling so much.

Another common technique for dealing with outliers is to use the intensity histogram to find the most popular intensity level in an image (i.e. the histogram peak) and then define a cutoff fraction which is the minimum fraction of this peak magnitude below which data will be ignored.

\section{UNSHARP FILTER}

The unsharp filter is a simple sharpening operator which derives its name from the fact that it enhances edges by subtracting blurred, unsharp or smoothed version of image from the image itself.

Unsharp masking produces an edge image $g(x, y)$ from an input image $f(x, y)$ via

$g(x, y)=f(x, y)-f_{\text {smooth }}(x, y)$ Eq...(2)

here $f_{\text {smooth }}(x, y)$ is a smoothed version of. $f(x, y)$ (See Figure 1.)

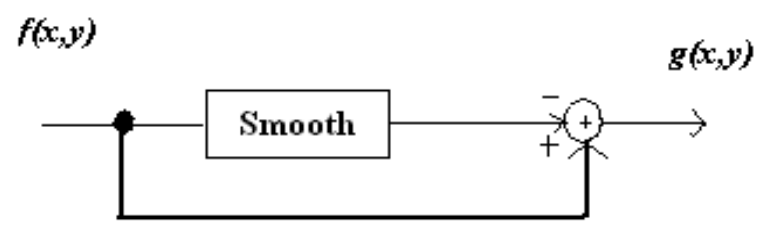

Fig 1 :Spatial sharpening.

We can better understand the operation of the unsharp sharpening filter by examining its frequency response characteristics. If we have a signal as shown in Figure 2(a), subtracting away the lowpass component of that signal yields the highpass, or 'edge', representation. Calculating an edge image for unsharp filtering[17].

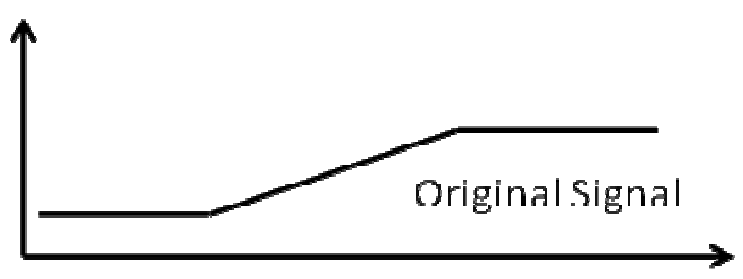

Fig 2 : Original Signal.

This edge image can be used for sharpening if we add it back into the original signal, as shown in Figure 3.

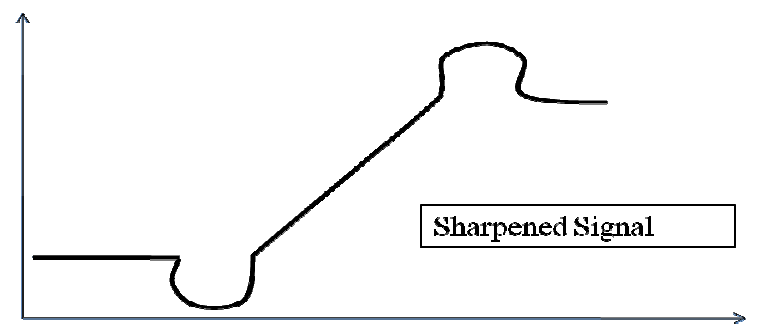

Fig 3 : Sharpening the original signal using the edge image.

Thus, the complete unsharp sharpening operator is shown in following Fig 4. 


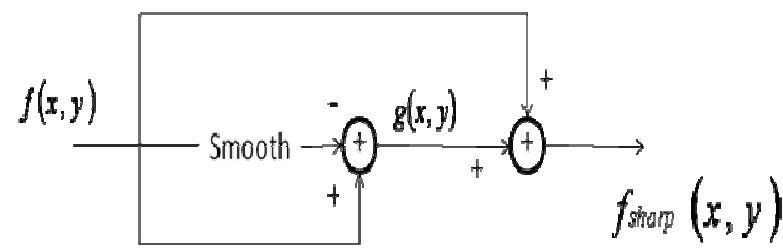

Fig 4 : The complete unsharp filtering operator.

We can now combine all of this into the equation: $f_{\text {sharp }}(x, y)=f(x, y)+k^{*} g(x, y)$ Еq...(3)

where $k$ is a scaling constant. Reasonable values for $k$ vary between 0.2 and 0.7 , with the larger values providing increasing amounts of sharpening. The unsharp filter is a powerful sharpening operator, but does indeed produce a poor result in the presence of noise.

\section{QUALITY MEASURES}

A good objective quality measure should well reflect the distortion on the image due to, for example, blurring, noise, compression, sensor inadequacy [18].

In this study we consider several image quality metrics and study their statistical behavior. The quality of image is measured by statistical mean, standard deviation \& histogram.

\subsection{Mean}

The arithmetic mean is the image's average value.

mean $=\sum\left(P_{x, y}\right) /(x * y)$ Eq...(4)

\subsection{The standard deviation $(\sigma)$}

It is a frequency distribution, or range of pixel values of an image. If an image is supposed to be uniform throughout, the standard deviation should be small. A small standard deviation indicated that the pixel intensities do not stray very far from the mean; a large value indicates a greater range. $\sigma=\left(\left(\sum\left(P_{x, y} * P_{x, y}\right) /(x * y)\right)-(\text { mean } * \text { mean })\right)^{1 / 2}$

In the above two equations $\sum\left(P_{x, y}\right)$ represents the summation of all pixel values in the image \& $\sum\left(P_{x, y} * P_{x, y}\right)$ is the sum of square of all pixel values.

When computing contrast values we usually used standard deviation because its dimensions are directly in terms of intensity values. These statistical values can be very accurate indicators of image quality \& may be used to make automated decisions.

\subsection{Image Histogram}

Histogram is an important digital image tool. It is statistical representation of the data within an image that shows how many pixels there are with each of the possible values. The histogram is a bar graph. Each vertical bar in the graph indicates the number of pixels of that value. The sum of all vertical bars is equal to the total number of pixels in the image[19]. This is never actually graphed but is processed by other programs to make decisions as to how to manipulate the image. Histogram represents statistical analysis of an image. It indicates the distribution of image data values using these values we can determine the following things:-

- The best contrast image.

- The overall brightness or darkness of an image. How to apply a function, such as thresholding to an image.

- We can judge the effectiveness of contrast enhancement, applied to an image.

\section{EXPERIMENT}

In this experiment the digital images of sputum sample are enhanced by using Contrast stretching and Unsharp Masking. The variation of results from these two techniques are shown here.

Followings are Microscopic digital images of Sputum sample for image analysis.

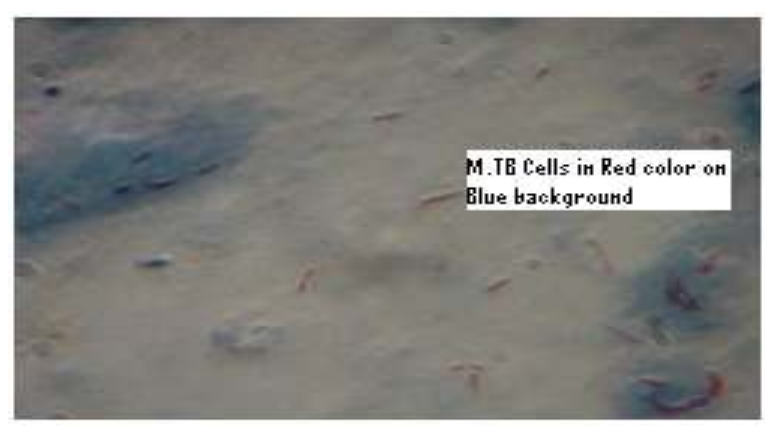

Fig 5 : Original image of Microscopic field of $\mathrm{ZN}$-Stain sputum smear on AFB slide.

The figure 5. is the original Microscopic image of ZN-Stained sputum smear under $100 \mathrm{X}$ lens captured by digital Microscope.

The image quality of original image is measure by using Mean, Std. deviation and Histogram. The Mean and Std. deviation are shown in the table 1 and the histogram of original image are shown in the following figure 6 .
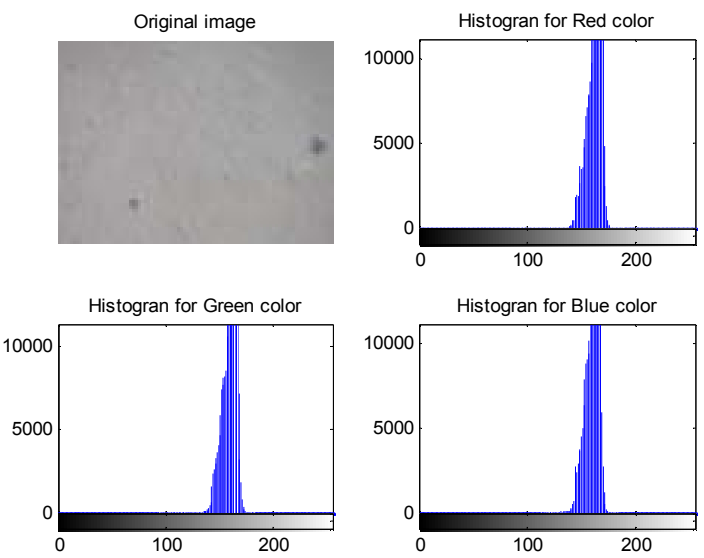

Fig 6 : Original image, Histogram for Red color for the original Image, Histogram of Green color for the original Image and Histogram of Blue color for the original Image. 


\subsection{Image Processed by contrast stretching}

Contrast stretching is applied on the original image the processed image is shown below in figure 7 . The image quality is measured in table 1 by using mean, standard deviation and results of histogram of processed image are shown in this figure 7. After applying Contrast stretching visual quality of the original image is enhanced by mean of visual perception. The histogram bars are spread uniformly as compare to original bars of histogram.
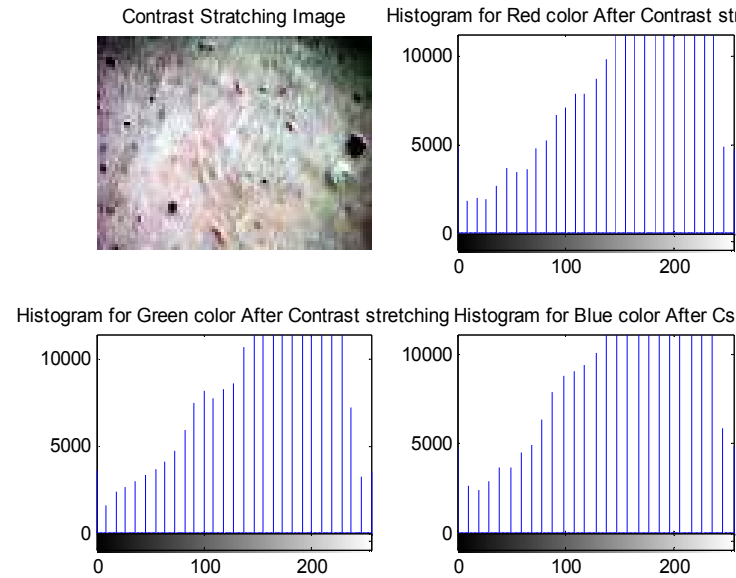

Fig 7 : Contrast stretching image, Histogram for Red color for the contrast stretching image, Histogram of

Green color for the contrast stretching image and Histogram of Blue color for the contrast stretching image.

\subsection{Image Processed by unsharp masking}

The image is sharpened by using unsharp masking which is shown in the following figure 8 . quality of these images are measured by using mean, standard deviation and histogram. The result of histogram is shown below.
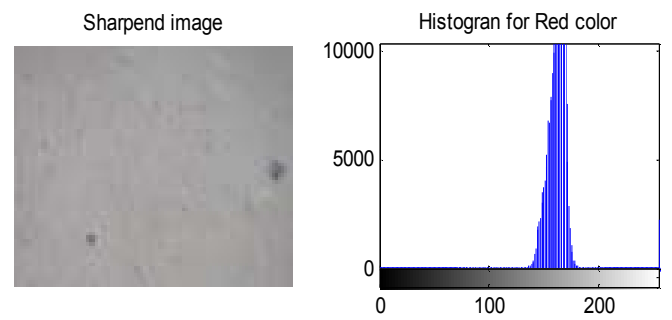
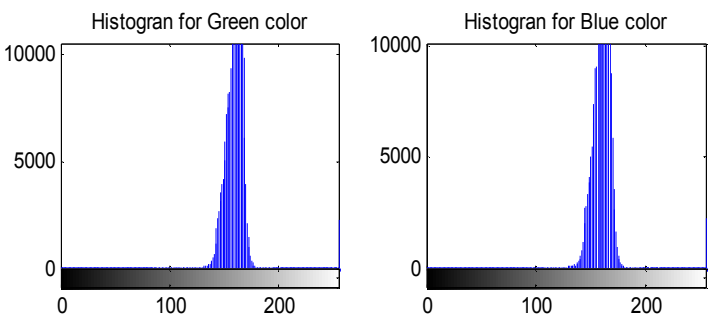

Fig 8 : Sharpened image, Histogram for Red color for the sharpened image, Histogram of Green color for the sharpened image and Histogram of Blue color for the sharpened image.

\section{COLOR SEGMENTATION}

Segmentation is a process that partitions an image into regions. Here we partitions image in two regions one for M.TB bacilli cells i.e. originally red in color thin rod shape region and other for background or part which is not in interest i.e. originally blue color region. In color imaging, each pixel is characterized by three RGB values. However, with multi spectral or multilayer images such as RGB image, it can be difficult to specify the selection criteria for segmentation. The main objective to filter out blue pixels and retain the reddish pixels which are the mycobacterium.

Here analysis of 34 images of sputum smear slide with ZhilNeelsen(Acid Fast Stain). For identification of M.TB cells for chromatic study we taken five pixels are from tuberculosis area i.e. from bacterial cell region i.e thin rod shape red region, and five pixels from sputum area i.e. blue background sputum region from each image. Calculate the mean for RGB intensities for each image. The color thresholding technique is carried out based on the color information of the bacterium to extract its images from the sputum and other objects. This technique specifies the range of RGB intensities for thresholding. The objects that lie outside the selection range will be rejected. Therefore, it is very important to determine the selection range because if this threshold cannot acquire a suitable value for RGB intensities, the thresholding algorithm will extract pixels other than the expected object.

The properties of the RGB pixels are being studied to extract the important features from the image. Based on the color information, the color thresholding algorithm should be able to extract the pixels of mycobacterium and reject pixels of other objects. In order to view the important properties of each segment so that necessary features and accurate value of threshold can be obtained from the image. The thresholding procedure must be done to the red, green and blue components.

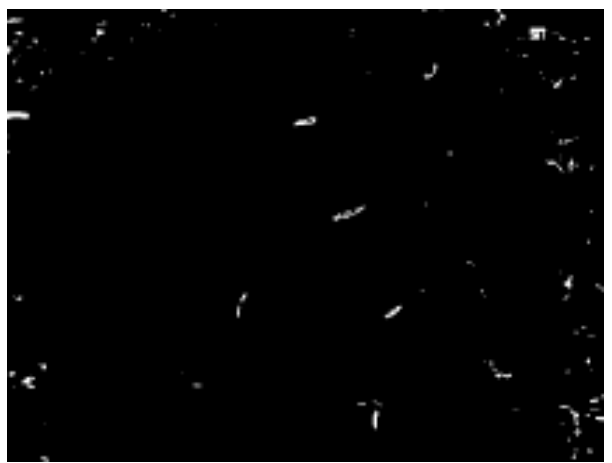

Fig 9 : Segmented Sharpened image of Microscopic field on AFB slide

After applying threshold algorithm on the image enhanced by Contrast stretching the figure 10 obtained. And on the image enhanced by unshap filter the figure 9 is generated as output. These segmented images are shows two values either 255 (white pixel) which indicate the area of M.Tb pixels or 0 (black pixel) sputum area. 


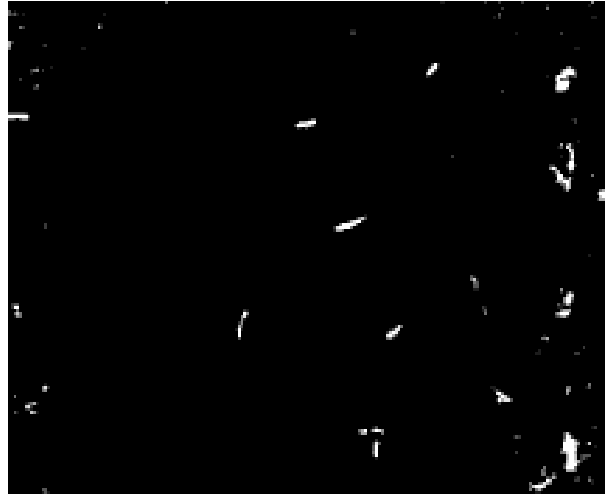

Fig 10 : Segmented Contrast stretching image of Microscopic field on AFB slide

We get final value is either 255 (white pixel) or 0 (blacke pixel) i.e M.TB cells are white in color on black background.

\section{RESULT AND DISCUSSION}

These are microscopic digital image of sputum smear dye by using Zhil-Neelson stain Known as Acid Fast stain (AFBAcid Fast bacilli). These images are enhanced for further process of Identification of bacilli (Mycobacterium Tuberculosis cells-M.TB Cells) from the sputum smear.

These digital images are studied by thresholding algorithm are brought through image enhancement process. This involves the adjustment of brightness, contrast and color in an image so that the pixel values fall into about the same range.

The enhancement is to be carried out by two technique contrast stretching and unsharp filter. By enhancing 34 images the quality measures mean and standard deviation are calculate shows in Table 1. observing these values the original image mean value is the lowest value then sharpened image mean value is little but incremented but the contrast stretching image mean value is the highest value. Similarly the original image standard deviation value is lowest value, then sharpened image std. deviation value \& contrast stretching image std. deviation value. And contrast stretching std. deviation value is the highest value than the sharpened image std. deviation value \& original image standard deviation value.

The histogram of these two techniques are shown in img.no.3 $\& 4$. The bars of histogram from contrast stretching image is uniformly distributed for red, green $\&$ blue color intensities than the bars of histogram from sharpened image for red green \& blue intensities.

The image segmentation carried out on RGB images enhanced by contrast stretching technique clearly identifies M.TB cells in figure 10. than the segmentation on sharpened images by mean of visual perception.

The quality measures of enhanced images shown in table no. 1 for analysis of 34 images. As per values obtained from the table 1. highest values of mean \& standard deviation are obtained from contrast stretching than unsharp filter. The following Bar chart also shows the contrast stretching image mean bars are longer than the original \& sharpened images mean bars. Similarly the standard deviation bars are longer than the original \& sharpened images bars. The histogram from contrast stretching images shows best contrast image. The overall brightness or darkness of an image uniformly distributed for RGB colors which is not observe in unsharp filtering. The segmentation carried on contrast stretching images are more accurately segment by mean of size, shape \& color manner so identification of the M.TB cells is more clearly than the sharpen image segmentation. Hence contrast stretching is more better than unsharp filter for the M.TB bacilli identification.

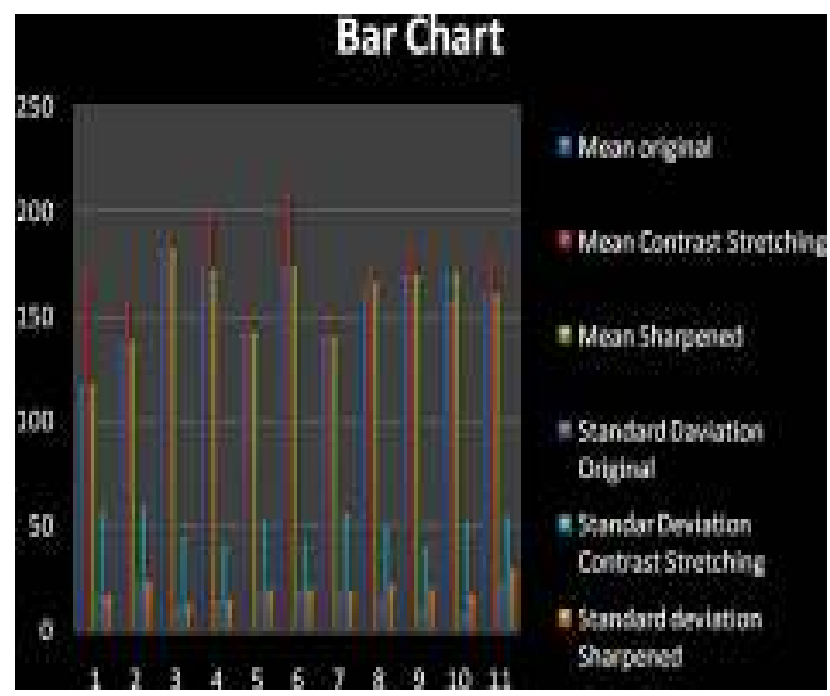

Graph 1 : Indicate Bar graph shows quality measures for ZN - Stain sputum smear digital images before and after enhancement using two techniques.

\section{CONCLUSION}

Contrast stretching i.e Normalization is commonly used to improve the contrast in an image without distorting relative level of intensities. By observing the bar chart that shows that contrast stretching image mean bars are longer than the original \& sharpened images mean bars. Similarly the standard deviation bars are longer than the original \& sharpened images bars. The histogram from contrast stretching images shows best contrast image. Visual perception of segmented images using contrast stretching is more clear than the segmentation using sharpen images. So the identification of the M.TB cells is more proper using contrast stretching. By observing all these measures \& from the result of segmentation it is concluded that contrast stretching is best enhancement technique than unsharp filter for the segmentation purpose \& M.TB bacilli identification.

\section{ACKNOWLEDGMENT}

Special thanks to Dr. Deshpande, TB Officer and Mr. Pawar, TB, Lab Technician, TB Hospital Aurangabad. 


\section{REFERENCES}

[1] WHO Report 2009,"Global tuberculosis Control a short update to the 2009 report".

[2] Module for MPWs and other DOT providers. Revised National Tuberculosis Control Program, Directorate general health and family welfare, Nirman bhavan New Delhi March 2006.

[3] J. A. C. Luna, "A Tuberculosis Guide for Specialist Physicians", International Union against Tuberculosis and Lung Disease,2004.

[4] K. Veropoulos, G. Learmonth, C. Campbell, B. Knight, and J. Simpson, "Automatic identification of tuberclebacilli in sputum. a preliminary investigation," Analytical and quantitative cytology and histology 21(4), pp. 277-281, 1999.

[5] K. Veropoulos, C. Campbell, G. Learmonth, B. Knight, and J. Simpson, "The automatic identification of tubercle bacilli using image processing and neural computing techniques," in Proceeding of the 8th international conference on artificial neural networks, 2, p. 797, 1998.

[6] M. Wilkinson, Fluorescence microscopy and fluorecent probes, ch. Rapid automatic segmentation of fluorescent and phase-contrast images of bacteria.

[7] J. Alvarez-Borrego, R. Mourino,G. Cristobal, and J. Pech, "Invariant optical color correlation for recognition of vibrio choleraeo1," in Int. conf. on pattern recognition, 2847, p. 283, (Barcelona, Spain), 2000.

[8] P. Demantova, D. Sakamoto, S. Ioshii, and H. Gamba, "Segmentacao autom'atica de bact'erias para o m'etodo deft," in Proceedings of the II latin american congress on biomedical engineering, (Havana, Cuba), 2001.

[9] M. Forero, E. Sierra, J. Alvarez-Borrego, J. Pech, G. Crist'obal, L. Alcal'a, and M. Desco, "Automatic sputum color segmentation for tuberculosis diagnosis," in Algorithms and systems for optical information processing, 2001.
[10] M. Forero, F. Sroubek, J. Alvarez-Borrego, N. Malpica, G. Crist'obal, A. Santos, L. Alcal'a, M. Desco,and L. Cohen, "Segmentation, autofocusing and signature extraction of tuberculosis sputum images," in Photonic devices and algorithms for computing, 2002.

[11] Manuel G. Forero, Gabriel Cristobal and Josue AlvarezBorrego "Automatic identification techniques of tuberculosis bacteria"

[12] R.A.A.Raof, ZalehaSalleh, S.I. Sahidan,M.Y. Mashor et,al "Color Thresholding Method For Image Segmentation Algorithm Of Ziehl-Neelsen Sputum Slide Images" 2008

[13] Vishnu Makkapati, Ravindra Agrawal and Raviraja Acharya "Segmentation and Classification of Tuberculosis Bacilli from ZN-stained", 5th Annual IEEE Conference on Automation Science and Engineering Bangalore, India, August 22-25, 2009

[14] Tuberculosis Training Module, Govt of Maharashrta.

[15] R. C. Gonzalez, and R. E. Woods, Digital Image Processing, Third Edition. Pearson Education, 3rd Ed., Copyright 2008.

[16] R. Fisher, S. Perkins, A. Walker, E. Wolfart (2003), "Contrast Stretching", http ://homepages.inf.ed.ac.uk/rbf/HIPR2/stretch.htm

[17] R. Fisher, S. Perkins, A. Walker, E. Wolfart (2003), "Unsharp filter", http://homepages.inf.ed.ac.uk/rbf/HIPR2/unsharp.htm

[18] Smail Avcibas, Bu“ lent Sankur, Khalid Sayood "Statistical evaluation of image quality measures", 206 / Journal of Electronic Imaging / April 2002 / Vol. 11(2)

[19] Image Statistics Based on material from Digital Imaging: Theory and Applications, H. E. Burdick, McGraw-Hill, 1997) 
Table 1: The table shows the statistical quality measures for digital images of $\mathrm{ZN}$-stain sputum smear images for identification of M.TB Bacilli cells

\begin{tabular}{|c|c|c|c|c|c|c|}
\hline \multirow{3}{*}{$\begin{array}{c}\text { Name } \\
1 \\
\end{array}$} & \multicolumn{3}{|c|}{ Mean } & \multicolumn{3}{|c|}{ Standard Deviation } \\
\hline & Original & $\begin{array}{l}\text { Contrast } \\
\text { Stretch } \\
\end{array}$ & Sharpened & Original & $\begin{array}{l}\text { Contrast } \\
\text { Stretch } \\
\end{array}$ & Sharpened \\
\hline & 161.526 & 167.8935 & 161.5597 & 8.6967 & 46.4862 & 17.4237 \\
\hline 2 & 163.674 & 169.5572 & 163.708 & 8.9067 & 48.116 & 17.549 \\
\hline 3 & 159.935 & 170.3907 & 159.9622 & 7.2944 & 52.5222 & 8.0719 \\
\hline 4 & 160.841 & 164.1402 & 160.8633 & 6.7473 & 164.1402 & 7.4549 \\
\hline 5 & 157.623 & 158.0837 & 157.6487 & 6.4821 & 54.2601 & 7.3419 \\
\hline 6 & 148.596 & 169.8752 & 148.6324 & 10.5972 & 50.2146 & 11.574 \\
\hline 7 & 148.449 & 149.0525 & 148.4812 & 7.4222 & 46.6895 & 8.5281 \\
\hline 8 & 155.374 & 168.7062 & 155.3982 & 7.2468 & 52.6256 & 8.0785 \\
\hline 9 & 154.266 & 164.3296 & 154.2978 & 8.343 & 52.6046 & 9.3017 \\
\hline 10 & 151.749 & 154.8472 & 151.7838 & 8.0367 & 49.2824 & 9.1308 \\
\hline 11 & 144.815 & 175.364 & 144.8475 & 8.7163 & 44.59 & 9.7744 \\
\hline 12 & 147.969 & 152.9283 & 148.0054 & 8.0806 & 50.1015 & 9.3791 \\
\hline 13 & 161.088 & 161.0748 & 161.1131 & 6.6553 & 57.1465 & 7.4485 \\
\hline 14 & 151.754 & 214.4112 & 151.7813 & 26.2696 & 53.0697 & 26.5093 \\
\hline 15 & 163.66 & 160.6373 & 163.6883 & 6.7775 & 49.3571 & 7.6387 \\
\hline 16 & 140.788 & 143.6182 & 140.8426 & 17.0498 & 51.5182 & 19.1089 \\
\hline 17 & 128.969 & 141.8517 & 129.0217 & 16.4144 & 52.7173 & 18.2376 \\
\hline 18 & 132.83 & 164.2679 & 132.8762 & 12.3293 & 57.09 & 13.7448 \\
\hline 19 & 93.1394 & 156.4346 & 93.193 & 13.9876 & 51.4859 & 15.5437 \\
\hline 20 & 116.342 & 171.235 & 116.3914 & 15.7583 & 56.6164 & 16.8203 \\
\hline 21 & 137.003 & 156.9344 & 137.0564 & 21.6392 & 58.7158 & 22.7703 \\
\hline 22 & 180.794 & 186.6762 & 180.8296 & 11.0977 & 43.6602 & 11.8693 \\
\hline 23 & 171.245 & 198.8925 & 171.2862 & 13.8439 & 40.3409 & 14.4953 \\
\hline 24 & 140.788 & 143.6182 & 140.8426 & 17.0498 & 51.5182 & 19.1089 \\
\hline 25 & 172.654 & 208.0974 & 172.6917 & 18.4145 & 40.6578 & 19.0505 \\
\hline 26 & 139.245 & 148.9451 & 139.2847 & 17.4997 & 55.0623 & 18.2271 \\
\hline 27 & 164.985 & 172.1093 & 165.0252 & 15.1824 & 49.254 & 21.7603 \\
\hline 28 & 169.035 & 181.9821 & 169.0352 & 10.2852 & 39.3987 & 19.2709 \\
\hline 29 & 169.917 & 155.4249 & 169.9505 & 7.0102 & 50.1862 & 16.7051 \\
\hline 30 & 159.307 & 176.1051 & 159.343 & 21.245 & 53.4279 & 28.8406 \\
\hline 31 & 164.392 & 180.8023 & 164.4184 & 16.2326 & 45.3421 & 24.2234 \\
\hline 32 & 161.561 & 172.0867 & 161.5905 & 10.3675 & 43.4001 & 20.9975 \\
\hline 33 & 156.136 & 160.3348 & 156.1721 & 7.6412 & 49.6643 & 17.7155 \\
\hline 34 & 153.145 & 197.9034 & 153.1792 & 15.4336 & 42.4051 & 23.7784 \\
\hline
\end{tabular}

